\title{
Uso de QR Code e Realidade Aumentada como suporte a visitação de museu
}

\author{
Uellisson Lopes da Silva, Robson Ferreira Braga, Daniel Scherer \\ Departamento de Computação - Universidade Estadual da Paraiba (UEPB) \\ Campina Grande - PB - Brazil \\ \{uellissonlopes, robson.f16, daniel.scherer\}@gmail.com
}

\begin{abstract}
This article presents a application developing proposal in augmented reality and in QR code, to support the visitation of a arts museum. The applications will allow Museum visitors will be able to access additional information about works on exposition, from a webcam and a computer monitor or with a mobile device.
\end{abstract}

Resumo. Este artigo apresenta a proposta de desenvolvimento de duas aplicações, uma usando Realidade Aumentada e outra QR code, para apoiarem a visitação de um museu de artes. Os aplicativos permitirão aos visitantes do museu, acessarem informações adicionais das obras de artes, no primeiro aplicativo, a partir de uma webcam e um monitor de computador que serão disponibilizados no local e no segundo com um celular ou tablet.

\section{Introdução}

A tecnologia esta cada dia mais presente na vida social das pessoas, de modo que ela está inserida em várias áreas tradicionais, como: educação, esporte, saúde, mercado financeiro, etc. Assim como essas áreas, a cultura não ficou de fora dos avanços tecnológicos. Nesse sentido temos como exemplo claro, o surgimento de vários museus virtuais, os quais podem ser visitados remotamente, por meio de um computador conectado a internet. Além do mundo virtual, também podemos citar várias experiências com tecnologia em museus reais, geralmente envolvendo recursos multimídia (áudio, vídeo e imagem), usados para apoio a visitação deste museus.

Considerando que os museus possuem diversas obras de arte, tais como quadros, esculturas e instalações artísticas, que lá estão para serem preservados em ambiente controlado e possibilitar ao público a oportunidade de observá-los; levando-se em conta ainda que um dos principais objetivos para exposições de obras vem a ser o cunho educacional, permitindo aos visitantes conhecerem tais obras, o uso das tecnologias pode ser interessantes no sentido de fornecer ao visitante, informações extras que facilitem a compreensão das obras ou mesmo para que o observador atente detalhes significativos. Tradicionalmente os museus adotam a figura do guia de visitas, funcionário treinado para guiar e apresentar informações aos visitantes, ou em alguns casos o uso de fones e aparelhos que possibilitam ouvir explicações sobre as obras (muitas vezes com a dispensa do guia). 
O uso de visitas guiadas possibilita uma gama interessante de interações, porém pode resultar em problemas, tais como: desconhecimento de todos os detalhes das peças expostas; fadiga após inúmeras visitações guiadas, podendo resultar em esquecimento de informações; número de guias necessários para que não haja uma espera demasiada entre um grupo e outro; desconhecimento ou mesmo inexperiência do guia em trabalhar com grupos que exijam outro idioma.

Já do ponto de vista de aparelhos sonoros e fones de ouvido, não há problema com a fadiga após inúmeras visitações guiadas, esquecimento de informações ou desconhecimento de detalhes, porém pode haver o problema de dificuldade de interação entre visitante e aparelho, que por sua vez pode resultar no observador olhar para uma obra e escutar as informações sobre outra.

No sentido de solucionar estas limitações e contribuir para um melhor aproveitamento das visitas aos museus, este artigo propõem o uso de QR code e RA para auxiliarem a disponibilização de informações extras sobre as obras de artes. Embora estas tecnologias exijam o desenvolvimento e instalação de aplicativos, até mesmo nos dispositivos do usuário (aplicativo em QR code), elas favorecem bastante a interatividade entre usuário e máquina, o que pode deixar mais sutil o uso da tecnologia, mantendo o foco na obra de arte. Além disso, tal proposta possibilita eliminar boa parte das dificuldades das formas de interação citadas, pois faz uso do próprio aparelho móvel da pessoa associado a uma interface mais dinâmica de uso (no caso de aparelhos com tela touch e o uso de $\mathrm{QR}$ code) ou mesmo de operar um totem com um monitor, para os casos de RA.

Desse modo, é apresentado um projeto em fase de execução, que tem como objetivo o desenvolvimento de duas aplicações, uma com $Q R$ code e outra com RA, que servirão de apoio a visitação do Museu de Artes Assis Chateaubriand, possibilitando que os visitantes possam ter acesso a informações extras sobre algumas das obras expostas, tanto em formato multimídia (imagem e vídeos), através da aplicação em $Q R$ code, quanto objetos virtuais $3 \mathrm{D}$, com o aplicativo em RA.

A próxima seção é composta por algumas considerações sobre as tecnologias usadas no projeto, a seguinte apresenta projetos relacionados. Na seção 4 será feita uma descrição sucinta da metodologia empregada no desenvolvimento do projeto, seguida das considerações finais e referências bibliográficas.

\section{Realidade Aumentada e QRCode}

A Realidade Virtual (RV) e a Realidade Aumentada (RA) são tecnologias recentes de interação entre homem e computador, que vêm se tornando mais populares a cada dia, movidas principalmente pelos avanços consideráveis no processamento dos microcomputadores e redução nos custos de aquisição dos equipamentos [Kirner e Tori, 2004].

Aplicativos que trabalham com RV, operam sobre um ambiente total e exclusivamente gerado por computador, e para uma completa imersão, demandam equipamentos específicos. Já a RA, também chamada de Realidade Misturada, incrementa o ambiente real com objetos virtuais [Kirner e Tori, 2004]. O usuário pode visualizar o ambiente de RA através de um capacete HMD (Head-Mounted Display), que combina diretamente elementos do mundo real com o virtual. Outra forma acontece 
através do rastreamento de marcadores que serão visualizados na tela de um computador ou dispositivos semelhantes (e.g.: tablets e celulares).

Pensando-se em termos educacionais, a possibilidade de se mesclarem objetos virtuais em ambientes reais permite uma interação que facilita a compreensão, pelo usuário, da relação entre concreto e o abstrato.

Uma das aplicações aqui propostas baseia-se na RA a partir do rastreamento de códigos impressos (marcadores), que são capturados por uma webcam e mapeados por um software, denominado toolkit [Kirner e Tori, 2004]. O fluxo de funcionamento de sistemas de RA, baseados em ARToolKit, pode ser observado na figura 1.

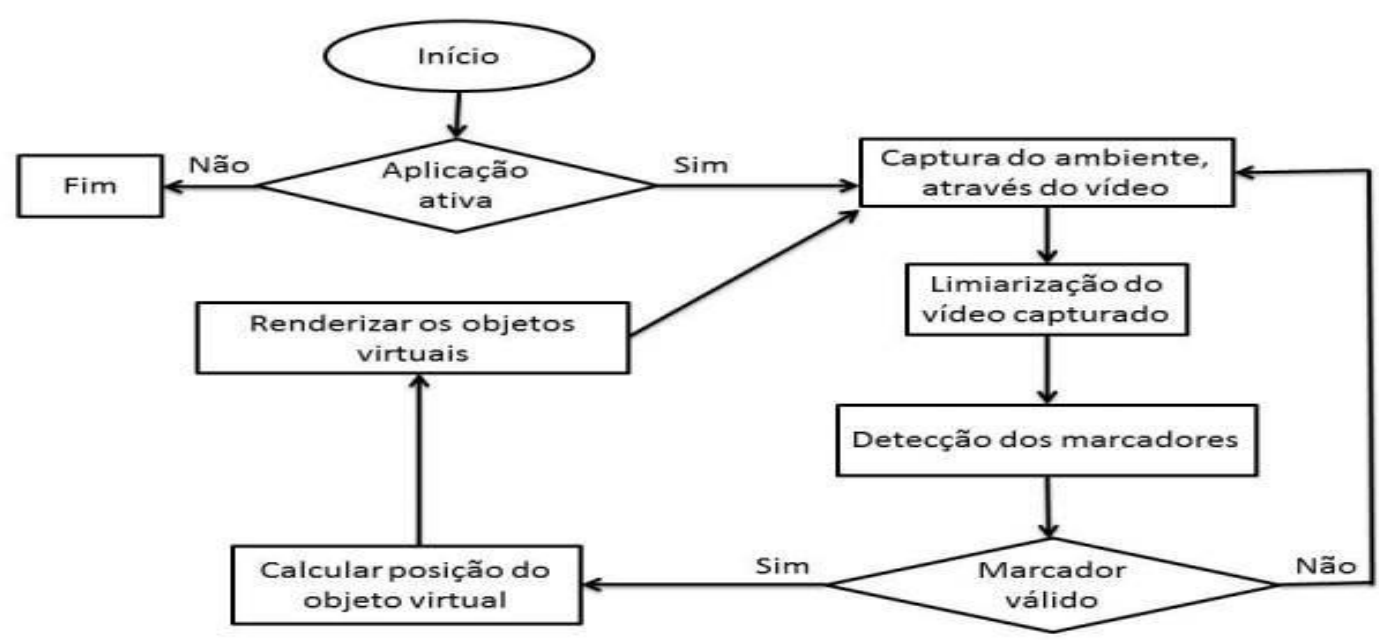

Figura 1. Fluxograma de funcionamento de sistemas de RA baseados no ARToolkit

A outra tecnologia usada neste projeto também se baseia no rastreamento de códigos impressos. O QR code (Quick Response Code ou código de resposta rápida) é uma espécie de simbologia desenvolvida pela Denso Wave em 1994 e utilizada para armazenar uma pequena quantidade de informações [QRcode.com, 2012]. Nesse projeto pretende-se trabalhar usando páginas web com conteúdo (imagem, vídeo ou texto) refente a obra exposta no qual o usuário terá acesso a seu endereço por meio do QRCode.

\section{Trabalhos Relacionados e Fundamentação da Proposta}

Existem diversos projetos e aplicações desenvolvidas utilizando RA e $Q R$ code buscando a preservação e valorização da cultura, tais como: museus virtuais [Falcão e Machado, 2011], sistemas que incrementam sítios arqueológicos [Papagiannakis, et al. 2002], aplicativos de apoio à visitação de museus [Silveira, et al. 2011], aplicativos para divulgação de eventos [Schwartz, 2012], aplicações para incentivo ao turismo [Silva, et al. 2011].

O projeto Lifeplus, desenvolvido pelo grupo de pesquisa Miralab, da universidade de Genebra na Suíça e financiado pela união Europeia consiste em um protótipo de sistema de RA usado para incrementar o sítio arqueológico de Pompéia, na Itália. O sistema (figura 2) permite mesclar ao ambiente real e objetos virtuais 
(personagens, flora e fauna) de modo a recriar cenas da antiguidade. Para tanto, os visitantes precisam utilizar um capacete HMD e fones de ouvido [Papagiannakis, et al. 2002].

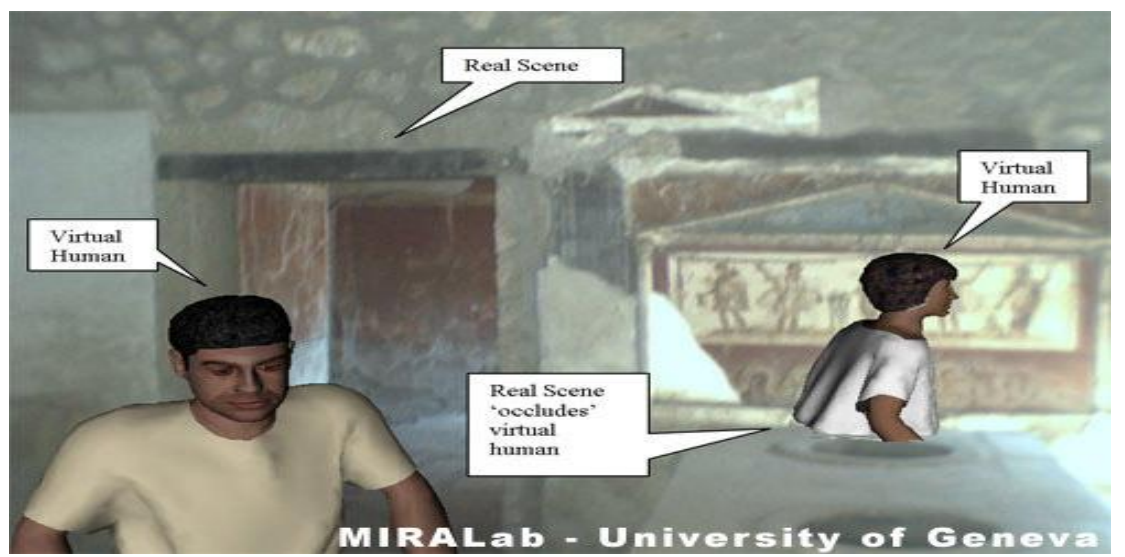

Figura 2: simulação de funcionais do sistema Lifeplus

Apesar da vantagem de imersão visual que o uso de um HMD proporciona, ela demanda que o usuário vista-se com o equipamento para poder fazer a visitação do sítio arqueológico, o que exige que o usuário consiga carregar todo o sistema. Além disto, há o custo financeiro considerável para a aquisição dos capacetes e a necessidade do pagamento da licença do software, que foi desenvolvido especificamente para o projeto.

O sistema Diálogos na Arte (figura 3) foi criado para registrar o discurso do público de um museu em relação aos objetos em exposição. Basicamente os visitantes podem fazer postagens de texto, imagem e vídeo sendo que, além da RA foram usadas outras técnicas, tais como: keycode (código alfa numérico) e geolocalização (a partir de posicionamento pelo GPS). No sistema foi colocado um marcador ao lado do objeto em exposição, para o qual o usuário aponta a câmera do tablet e consegue visualizar as postagens de outros visitantes que também viram a obra [Silveira, et al. 2011].

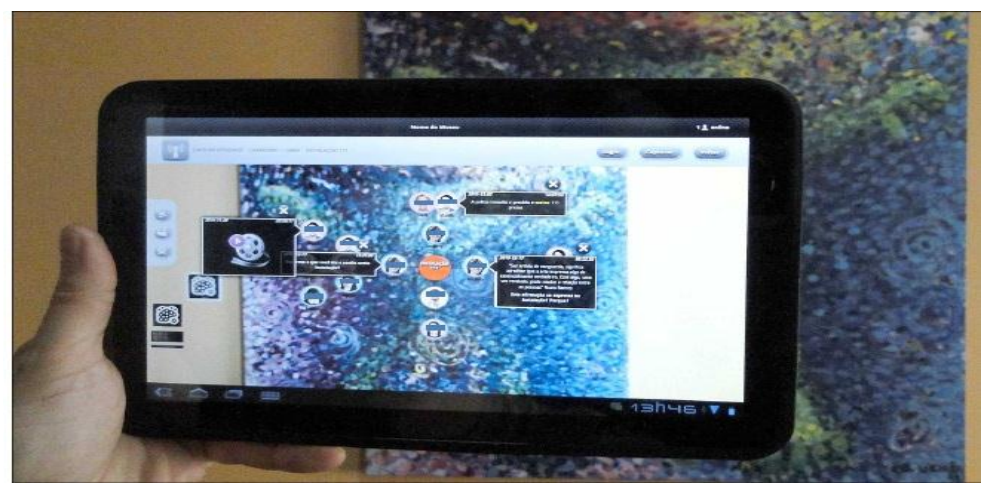

Figura 3 - demonstração do sistema Diálogos na Arte

Esse tipo de aplicação é interessante, por possibilitar que o visitante do museu possa ver detalhes que foram percebidos por outras pessoas. Porém, o uso indiscriminado acaba por resultar em spam, com surgimento de postagens sem (ou com pouca) credibilidade. No caso da proposta apresentada neste artigo, almeja-se que o visitante visualize materiais produzidos por especialistas, resultando em informações educacionalmente relevantes. 
Outro exemplo de uso de aplicativo com RA voltado para linha móvel em museus é o James May's Science Stories, no qual o apresentador de programa de televisão James May (transformado em um avatar), narra fatos sobre os objetos em exposição [Wired, 2012]. O usuário que estiver visitando o museu da ciência em Londres, ou até mesmo em casa, pode comprar o aplicativo e utilizá-lo em dez locais diferentes na galeria "Making of the Modern World" [Google Play, 2012]. Basicamente deve-se apontar a câmera do aparelho móvel para um dos marcadores presentes no local e o aplicativo reconhece a área onde será projetado o avatar de James. O aplicativo está disponível no Google Play e no iTunes e custa cerca de \$3 dólares.

Esse tipo de aplicativo está relacionado com a linha de desenvolvimento em RA desse trabalho. Entretanto, diferente da proposta do museu de Londres que apresenta um guia virtual para dar explicações ao visitante, o projeto aqui apresentado busca fornecer um materiais nos quais seja possível identificar detalhes extras das obras que, vistos por olhos destreinados, passariam despercebidos.

Um exemplo de aplicação utilizando QR Code e RA foi desenvolvido em Cuiabá-MS, visando a Copa do Mundo de Futebol em 2014, em especifico nesta cidadesede. O trabalho descreve a criação de um guia de turismo para celular que usa estas duas tecnologias. Para desenvolvimento do aplicativo foram utilizadas a API Google Chart Tools, para geração dos códigos 2D e a ferramenta FlarToolKit, para criação do ambiente de RA. O aplicativo deve ser usado em um cartão que contém um QR Code e um marcador de RA, sendo que para o primeiro o aplicativo informa a localização geográfica do ponto turístico e o segundo mostra um objeto 3D para visualização do mesmo [Silva, et al. 2011].

A proposta deste protótipo é bastante similar a linha de desenvolvimento deste artigo, porém espera-se que haja um melhor aproveitamento do uso do QR code, principalmente para apresentação de informações multimídia ao visitante.

Outro exemplo de QR Code é o da Revista da Imagem (Schwartz 2012), no qual a tecnologia foi utilizada para mostrar a programação do evento ICR 2012 (Congresso Internacional de Radiologia).

Diante disso, o projeto aqui apresentado tem como objetivo o desenvolvimento de dois aplicativos, um utilizando QR code e outro RA, para o Museu de Artes Assis Chateaubriand, com o objetivo de oferecer aos visitantes informações adicionais sobre as obras de arte, disponibilizadas na forma de texto, imagem, áudio, vídeo ou animações 3D. Qualificando ainda mais as visitas ao museu.

No caso do aplicativo com QR code, o usuário acessará as informações por meio de um celular ou tablet, que possua uma câmera e um leitor desse código previamente instalado. De modo que ao posicionar a câmera do dispositivo móvel para o marcador, ele será redirecionado para uma página web com informações sobre a obra, tal como vídeos curtos (até 3 minutos), imagens ou textos curtos (até 250 palavras) sobre as obras.

Já para a aplicação em RA, devido ao custo de equipamentos mais moderno e limitações quanto ao rastreamento dos marcadores e estabilidade das imagens apresentadas, inicialmente os visitantes irão interagir por meio de um totem composto por monitor equipado com uma webcam. Posteriormente, almeja-se o desenvolvimento da aplicação para uso também em dispositivos móveis. 


\section{Metodologia de desenvolvimento}

Partindo da definição da proposta, foi realizada uma pesquisa de modo a identificar a ferramenta de RA (toolkit) que servirá de base para o projeto [Braga et al. 2012].

Nesse sentido foi selecionada inicialmente o FLARAS (Flash Augmented Reality Authoring System), uma ferramenta de autoria desenvolvida a partir do FLARToolKit, que além de oferecer suporte ao desenvolvimento de aplicativos que podem ser executados em qualquer computador que possua um navegador com plug-in Flash Player, também permite visualização de texturas (imagem 2D), vídeos, objetos 3D ou áudios [Souza, R. C., et al. 2012]. Essa ferramenta possibilita salvar um arquivo, que poderá ser editado posteriormente. Permite também exportar o arquivo, criando um aplicativo que pode ser executado em qualquer navegador com plug-in Flash Player devidamente instalado [Souza, R. C., et al. 2012].

Originalmente, o projeto buscava o uso de RA tanto para apresentação de informações estáticas (textos) quadro dinâmicos (vídeos), além de aplicá-lo na visualização de imagens 3D. Neste sentido, foram realizados testes no FLARAS, buscando identificar limitações que pudessem prejudicar o andamento do projeto, tais como: requisitos de hardware (necessidade de webcam com resolução acima da média ou exigência de um alto poder de processamento dos computadores), dificuldades de rastreamento dos marcadores ou problemas de execução em diferentes sistemas operacionais; foram realizados testes de visualização de imagens, vídeos e objetos 3D (com e sem áudio).

Para testar a inserção de imagens (figura 4), forma desenvolvidos aplicativos usando texturas em diferentes formatos e resoluções, de modo a identificar a melhor opção para exibir informações textuais, gráficas (de baixa e alta resolução). A partir dos testes, percebeu-se em um primeiro momento que a qualidade da imagem se mostra mais relevante que o seu formato. O FLARAS suporta os seguintes formatos: JPG, PNG e GIF, sendo que:

- O formato JPG se mostra mais eficiente para imagens detalhadas, geralmente as que apresentam uma grande combinação de cores (ex.: fotografias);

- O formato PNG se mostra mais eficiente para imagens menos detalhadas (ex.: textos);

- Já o formato GIF, segundo [Suporte Microsoft, 2012], não é muito indicado, pois apresenta qualidade muito inferior aos dois anteriores.

Em relação a inserção de vídeo nessa ferramenta, também foram desenvolvidos alguns testes usando vídeos nos dois formatos suportados (FLV e MP4) e em diferentes resoluções. A partir destes testes, foi possível constatar que a ferramenta funciona razoavelmente bem com esse tipo de recurso, porém alguns aplicativos quando executados no navegador, apresentaram problemas de exibição, como presença apenas do áudio ou uma traja preta sobre o marcador após tirar o vídeo da cena, pelo que foi pesquisado esse problema está relacionado ao plug-in Flash Player ou ao navegador e não a ferramenta. Desse modo, é importante realizar vários testes de um aplicativo dessa natureza, sobretudo pelo fato deste plug-in ter sido descontinuado. 

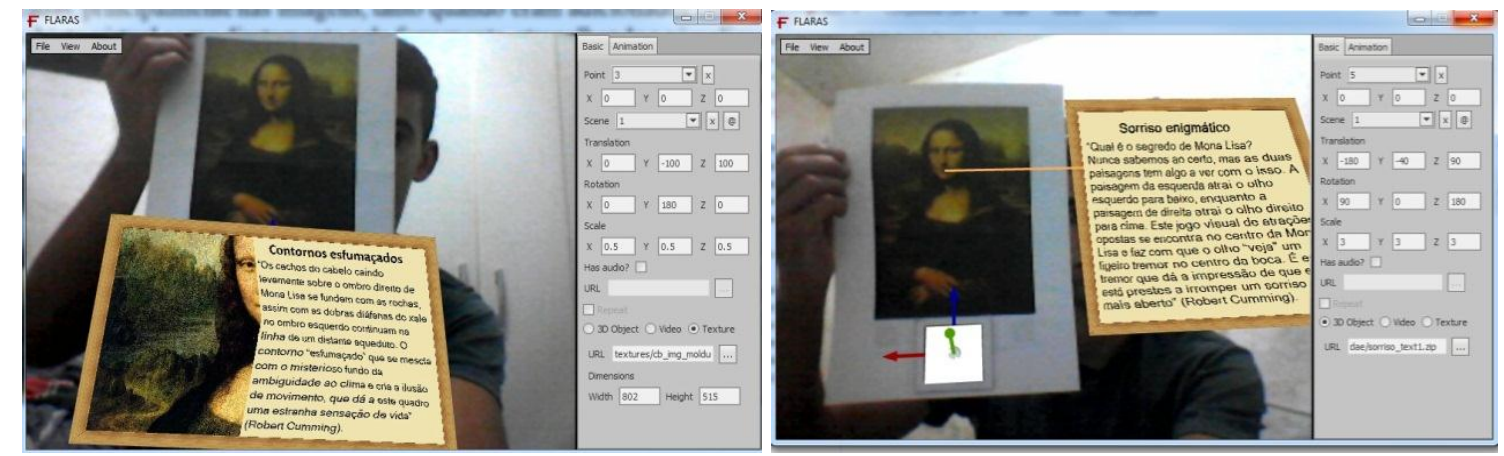

Figura 4: Protótipo usando imagens e objetos virtuais com textura no FLARAS

A partir dos testes do FLARAS foram identificados alguns problemas relacionados a visualização das informações, principalmente nas imagens com texto (figura 4). Desta forma, o uso do QR code foi identificado como uma alternativa, pois permite acesso rápido a informações multimídia, através de URL's associadas a eles, sem apresentar distorções. Além disto, tem-se um baixo custo financeiro, pois existem várias ferramentas gratuitas de geração e leitura desses códigos, inclusive para dispositivos móveis (smartphone e tablet), dependendo basicamente de um ambiente que disponibilize internet [Schwartz, 2012].

O uso de RA foi reformulado para permitir interação com peças que necessitem de modelos em 3D para facilitar sua compreensão (e.g.: uma escultura).

\subsection{Proposta de validação}

A validação será baseada em identificação da satisfação do usuário, tendo como parâmetros os definidos no protocolo proposto por [Aguiar et al, 2012] e seguindo os seguintes passos:

$1^{\circ}$ passo: reunir uma turma, entre cinco e dez alunos, de um curso graduação da UEPB, relacionado à área de artes (Filosofia ou História). Importante haver uma parcela de alunos que já visitaram o museu e outros que não visitaram, de modo a identificar diferenças de satisfação nos dois grupos;

$2^{\circ}$ passo: explanar rapidamente acerca do funcionamento dos aplicativos e do totem para visualização do modelo 3D;

$3^{\circ}$ passo: solicitar a autorização dos usuários para que a atividade e a entrevista sejam gravadas para fins de análise posterior;

$4^{\circ}$ passo: entregar o termo de sigilo da equipe de desenvolvimento, indicando que todas as gravações serão utilizadas para fins acadêmicos de análise do produto;

$5^{\circ}$ passo: permitir que os estudantes interajam livremente, dois a dois, com os aplicativos. Com a anuência dos usuários, a utilização deverá ser gravada digitalmente para posterior analise da usabilidade.

$6^{\circ}$ passo: realizar uma entrevista guiada por um questionário de satisfação de uso do software. Caso haja anuência do usuário, a entrevista será gravada para posterior análise;

$7^{\circ}$ proceder a análise do material gravado e dos questionários respondidos. 


\section{Considerações finais e resultados esperados}

Nesse artigo foi apresentada uma proposta de desenvolvimento de duas aplicações: uma com QR code e outra com RA; que apoiarão a visitação do Museu de Artes Assis Chateaubriand, permitindo disponibilizar informações extras sobre suas obras, que poderão ser visualizadas através de um dispositivo móvel celular ou tablet (QR code) ou de um totem composto por uma webcam e um monitor (RA).

Até o presente momento foi escolhido o software Quickmack para trabalhar com QR code, por ser gratuito e permitir a geração e leitura do marcador tanto em computador quanto por um celular ou tablet [Quickmack.com, 2012]. No caso do aplicativo em RA foi definido o toolkit FLARAS para o desenvolvimento das aplicações.

Até o momento foram desenvolvidos alguns protótipos que serviram de base para testes de funcionalidade e identificação de problemas no uso de RA para apresentação de informações textuais ou mesmo de vídeos, principalmente devido a distorções ocorridas pelo manuseio da webcam pelo usuário. Já para a visualização de objetos 3D, o RA tem se mostrado uma alternativa viável.

Quanto ao QR code, foram feitos alguns testes preliminares que permitiram identificar que não há problemas com distorções nos textos e vídeos. Além disto, foi iniciado o processo para testes no museu, onde será verificada a satisfação do usuário. Outro teste de validação esperado para o protótipo no museu vem a ser o comportamento da aplicação em ambientes que possuem sistemas, tipos e intensidades de iluminação diferenciadas.

\subsection{Próximas etapas}

Uma vez que já foram definidas as tecnologias, estão em processo de desenvolvimento os dois protótipos a serem implantados no museu, que serão validados a partir metodologia citada anteriormente. Além disto, tem-se: móveis;

- o desenvolvimento da aplicação em RA para ser utilizada em dispositivos

- desenvolvimento da aplicação utilizando QR code de modo que funcione totalmente off-line, buscando situações em que não haja a disponibilidade de Internet ou mesmo que isto gere um custo desnecessário;

\section{Agradecimentos}

Os autores agradecem pelo apoio financeiro na forma de bolsa CNPQ e UEPB de iniciação científica.

\section{Referências}

AGUIAR, Y. P. C. ; VIEIRA, M. F. Q ; GALY, E. ; MERCANTINI, J-M ; SANTONI, Charles . Refining a user behaviour model based on the observation of emotional states. In: Third International Conference on Advanced Cognitive Technologies and Applications - COGNITIVE, 2011, Rome. Proceedings of the Third International Conference on Advanced Cognitive Technologies and Applications, 2011. 
Braga, R.F., Lopes, U., Iwano, T.M., Scherer,D.. Estudo comparativo de toolkits de Realidade Virtual e Aumentada visando aplicação educacional. Desafie 2012. CSBC 2012.

Google Play. "James May's Science Stories". 2012. https://play.google.com/store/apps/details?id=com.digicave.beta.sciencestories. (acesso em: 22 de Julho 2012).

Kirner, Claudio, e Romero Tori. "Fundamentos e Tecnologias de Realidade Virtual e Aumentada.” VIII Symposium on Virtual Reality. 2004.

QRcode.com. “About QR Code”. 2012. http://www.qrcode.com/en/aboutqr.html. (acesso em: 20 de Setembro 2012).

Quickmack.com. "About”. 2012. http://www.quickmark.com.tw/en/basic/profile.asp. (acesso em: 20 de Setembro 2012).

Papagiannakis, G., Ponder M., Molet, T., kshirsagar, S., Cordier, F., Magnenatthalmann, N. e Thalmann, D. "LIFEPLUS: Revival of life in ancient Pompeii". 8th Int Conf. On Virtual Systems and Multimedia, 2002.

Silveira, A. L. M., Biazus, M. C.V. e Axt, M. "Diálogos na Arte: Sistema de Autoria em Realidade Aumentada." 22 ${ }^{\circ}$ Simpósio Brasileiro de Informática na Educação. Aracaju, 2011. 415-425.

Souza, R. C., Moreira, H. D. F., Kirner, C. "FLARAS 1.0 - Flash Augmented Reality Authoring System". 2012. http://ckirner.com/flaras/documentacao.html. (acesso em 30 de 07 de 2012).

Suporte Microsoft. "Diretrizes para selecionar o formato de imagem apropriado". http://support.microsoft.com/kb/272399/pt-br. (acesso em 10 de 07 de 2012).

Wired. "Top Gear Host Narrates Museum Exhibits - as Augmented Reality Avatar". 2012. http://www.wired.com/gadgetlab/2012/04/top-gear-host-narrates-museumexhibits-as-augmented-reality-avatar/. (acesso em: 22 de 07 de 2012).

Falcão, Eduardo L. e Machado, Liliane S. "Museu 3I: Publicação e Visitação Online de Acervos Tridimensionais”. VII Workshop de Realidade Virtual e Aumentada. 2010.

Schwartz, Rubens. "Facilitando por códigos". 2012. http://www.spr.org.br/files/public/periodical/public_245/versao_completa.pdf. (acesso em: 24 de 09 de 2012).

Silva, P. C. Neto, Nunes, C. e Nunes, E. P. dos Santos. "Integrando Recursos de Realidade Aumentada e Código de Barras Bidimensionais no Desenvolvimento de um Guia de Turismo". In WRVA 2011. 2011. 